\title{
A NOTE ON RECURSIVE CALCULATIONS OF PARTICULAR $9 j$ COEFFICIENTS
}

\author{
J.-C. Pain \\ Commissariat à l'énergie atomique et aux énergies alternatives, DAM, DIF, F-91297 Arpajon, France \\ E-mail: jean-christophe.pain@cea.fr
}

Received 24 May 2011; revised 27 June 2011; accepted 21 September 2011

\begin{abstract}
The calculation of angular-momentum coupling transformation matrices can be very time consuming and alternative methods, even if they apply only in special cases, are helpful. We present a recursion relation for the calculation of particular $9 j$ symbols used in the quantum theory of angular momentum.
\end{abstract}

Keywords: angular momentum, $9 j$ coefficient, recursion relation

PACS: 02.70.-c, 31.15.-p, 03.65.Fd, 23.40.-s

The coupling of $N$ angular momenta is related to the definition of a $3(N-1) j$ symbol. The $9 j$ symbols, characterizing the coupling of four angular momenta, are involved for instance in the computation of the matrix elements of the products of tensor operators, and in the transformation from $L S$ to $j j$ coupling [1, 2]. The triple sum series of Jucys and Bandzaitis [3] is the simplest known algebraic form for the $9 j$ coefficient. Fourty years ago, Ališauskas and Jucys [4-8] derived an algebraic expression, in which $9 j$ symbols are written as the threefold summation of multiplications and divisions of factorials. More recently, Wei [9, 10] proposed to express $9 j$ symbols as a summation of the products of binomial coefficients and devised an algorithm to calculate the binomial coefficients recursively. Although some relationships between particular $9 j$ symbols have been obtained in special cases [11], it is quite difficult to find recursion formulas for the $9 j$ symbol itself, due to the fact that it contains nine arguments.

The general recursion relations for arbitrary $9 j$ symbols were introduced in [12], [3] (with incorrect phase factors), and in [13] (in corrected form). However, as a rule in these relations, several angular momentum parameters are changing. It seems that relations with changing single momentum parameter are possible only if some other parameters of the $9 j$ accept extreme or fixed small values. For instance, the following $9 j$ symbol:

$$
\left\{\begin{array}{ccc}
\ell_{1} & \ell_{2} & L \\
j_{1} & j_{2} & L \\
1 / 2 & 1 / 2 & 1
\end{array}\right\}
$$

is often encountered in atomic physics and plays a major role in nuclear physics, especially for the study of $\beta$-decay [14, 15]. It can be evaluated in terms of $3 j$ symbols [13]:

$$
\begin{aligned}
\left\{\begin{array}{lll}
\ell_{1} & \ell_{2} & L \\
j_{1} & j_{2} & L \\
1 / 2 & 1 / 2 & 1
\end{array}\right\}= & \frac{1}{\sqrt{6(2 L+1)\left(2 j_{1}+1\right)\left(2 j_{2}+1\right)}} \\
& \times \frac{\left(\begin{array}{ccc}
\ell_{1} & \ell_{2} & L \\
1 / 2 & 1 / 2 & -1
\end{array}\right)}{\left(\begin{array}{lll}
L & j_{1} & j_{2} \\
0 & 0 & 0
\end{array}\right)}
\end{aligned}
$$

but the latter relation is verified only if $j_{1}, j_{2}$, and $L$ are integers so that $j_{1}+j_{2}+L$ is even [13, 16]. More generally, algebraic expressions for $9 j$ symbols

$$
\left\{\begin{array}{ccc}
\ell_{1} & \ell_{2} & L \\
j_{1} & j_{2} & L^{\prime} \\
1 / 2 & 1 / 2 & 1
\end{array}\right\}
$$


are tabulated as twelve entries of table 10.3 of Ref. [13], for

$$
\left\{\begin{array}{ccc}
a+\lambda b+\mu c+\nu \\
a & b & c \\
1 / 2 & 1 / 2 & 1
\end{array}\right\} .
$$
for

Moreover, in Appendix 5 of Ref. [3], only two entries

$$
X(b, d, f)=\left\{\begin{array}{ccc}
b+p d+q f+r \\
b & d & f \\
\beta & \delta & \phi
\end{array}\right\}
$$

with $\beta=\delta=1 / 2, \phi=1, p=q=1 / 2$ and, respectively, $r=0$ or $r=1$ are included, when the remaining ten entries of table 10.3 of Ref. [13] with $p= \pm 1 / 2, q= \pm 1 / 2, r=0, \pm 1$ can be generated using some compositions of the "mirror reflection" substitutions $b \rightarrow \bar{b}=-b-1, d \rightarrow \bar{d}=-d-1, f \rightarrow \bar{f}=$ $-f-1$ (see Eqs. (П.5.2) to (П.5.8) in Appendix 5 of Ref. [3]).

Special $9 j$ coefficients under consideration include three or four stretched triplets of angular momentum parameters [17]. In particular, eight entries of table 10.3 of Ref. [13] with $L^{\prime}=L \pm 1$ are expressed without sum by means of the following expression [3, 13]:

$$
\begin{aligned}
& \left\{\begin{array}{lc}
a b & a+b \\
d e & f \\
g h a+b+f
\end{array}\right\}= \\
& (-1)^{a+d-g} \frac{\Delta(a+b+f g h)}{\Delta(a d g) \Delta(b e h) \Delta(d e f)} \\
& \times\left[\frac{(2 a) !(2 b) !(2 f) !}{(2 a+2 b+1)(2 a+2 b+2 f+1) !}\right]^{1 / 2} \\
& \times \frac{(a+b+g+h+f+1) !(g-a+d) !}{(g+h-a-b-f) !(a+g+d+1) !} \\
& \times \frac{(e-b+h) !(d+e-f) !}{(b+e+h+1) !(d+e+f+1) !},
\end{aligned}
$$

where

$$
\Delta(a b c)=\left[\frac{(a+b-c) !(a-b+c) !(-a+b+c) !}{(a+b+c+1) !}\right]^{1 / 2},
$$

together with different symmetry properties of $9 j$ symbols. The following useful relation was mentioned by Jang [18]:

$$
A_{j_{1}, j_{2}}(L)\left\{\begin{array}{lll}
\ell_{1} & \ell_{2} & L \\
j_{1} & j_{2} & L+1 \\
1 / 2 & 1 / 2 & 1
\end{array}\right\}=
$$

$$
\begin{aligned}
& B_{\ell_{1}, \ell_{2}, j_{1}, j_{2}}(L)\left\{\begin{array}{lll}
\ell_{1} & \ell_{2} & L \\
j_{1} & j_{2} & L \\
1 / 2 & 1 / 2 & 1
\end{array}\right\} \\
& +C_{j_{1}, j_{2}}(L)\left\{\begin{array}{lll}
\ell_{1} & \ell_{2} & L \\
j_{1} & j_{2} & L-1 \\
1 / 2 & 1 / 2 & 1
\end{array}\right\},
\end{aligned}
$$

with $S=j_{1}+j_{2}+L$, and where

$$
\begin{aligned}
& A_{j_{1}, j_{2}}(L)=\left[L(2 L+3)(S+2)\left(S-2 j_{1}+1\right)\right]^{1 / 2} \\
& \times\left[\left(S-2 j_{2}+1\right)(S-2 L)\right]^{1 / 2} \\
& B_{\ell_{1}, \ell_{2}, j_{1}, j_{2}}(L)= \\
& (2 L+1)\left[\bar{j}_{1}-\bar{j}_{2}-\bar{L}+\frac{2 \bar{L}\left(\bar{j}_{2}+3 / 4-\bar{\ell}_{2}\right)}{\bar{\ell}_{1}+\bar{j}_{2}-\bar{j}_{1}-\bar{\ell}_{2}}\right]
\end{aligned}
$$

and

$$
\begin{aligned}
& C_{j_{1}, j_{2}}(L)=\left[(L+1)(2 L-1)(S+1)\left(S-2 j_{1}\right)\right]^{1 / 2} \\
& \times\left[\left(S-2 j_{2}\right)(S-2 L+1)\right]^{1 / 2} .
\end{aligned}
$$

We follow the convention of Biedenharn et al. [19], $\bar{x}=x(x+1)$. Formula $(8)$ is a particular case of the general recursion relations for $9 j$ symbols $[3,12,13]$. Each $9 j$ symbol depends on $L$ via two of its arguments. Defining for instance $u_{L}=\left\{\begin{array}{ccc}\ell_{1} & \ell_{2} & L \\ j_{1} & j_{2} & L+1 \\ 1 / 2 & 1 / 2 & 1\end{array}\right\}$, we have $u_{L-1}=\left\{\begin{array}{ccc}\ell_{1} & \ell_{2} & L-1 \\ j_{1} & j_{2} & L \\ 1 / 2 & 1 / 2 & 1\end{array}\right\}$, which does not appear in Eq. (8), except if $\left(j_{1}, j_{2}\right)$ and $\left(\ell_{1}, \ell_{2}\right)$ are interchanged. However, one has

$$
\left\{\begin{array}{ccc}
\ell_{1} & \ell_{2} & L \\
j_{1} & j_{2} & L+1 \\
1 / 2 & 1 / 2 & 1
\end{array}\right\}=
$$$$
\left[\frac{2 L+1}{L+\left(\ell_{1}-j_{1}\right)\left(2 \ell_{1}+1\right)+\left(\ell_{2}-j_{2}\right)\left(2 \ell_{2}+1\right)}-1\right]
$$$$
\times\left[\frac{L(2 L-1)(S-2 L)}{(L+1)(2 L+3)(S-2 L+1)}\right]^{1 / 2}
$$

$$
\times\left[\frac{\left(S-2 j_{1}+1\right)\left(S-2 j_{2}+1\right)(S+2)}{\left(S-2 j_{1}\right)\left(S-2 j_{2}\right)(S+1)}\right]^{1 / 2} \times
$$




$$
\times\left\{\begin{array}{ccc}
\ell_{1} & \ell_{2} & L \\
j_{1} & j_{2} & L-1 \\
1 / 2 & 1 / 2 & 1
\end{array}\right\},
$$

which can be combined to Eq. (8) to obtain a relation between the coefficients $\left\{\begin{array}{lll}\ell_{1} & \ell_{2} & L \\ j_{1} & j_{2} & L+1 \\ 1 / 2 & 1 / 2 & 1\end{array}\right\}$ and $\left\{\begin{array}{ccc}\ell_{1} & \ell_{2} & L \\ j_{1} & j_{2} & L \\ 1 / 2 & 1 / 2 & 1\end{array}\right\}$

Using the Regge symmetry of Wigner $3 j$ symbols [20], for $\ell_{1}-j_{1}=\ell_{2}-j_{2}= \pm 1 / 2$ (separately for both + and - signs) [3, 13], one finds, concerning the two other $9 j$ symbols involved in Eq. (8), that in some particular cases, as for the $9 j$ symbol of Eq. (1), explicit formulas in terms of $3 j$ symbols do exist [16, 21]:

$$
\begin{aligned}
& \left\{\begin{array}{lll}
\ell_{1} & \ell_{2} & L \\
j_{1} & j_{2} & L \\
1 / 2 & 1 / 2 & 1
\end{array}\right\}= \\
& \frac{\left(j_{1}-\ell_{1}\right)\left(2 \ell_{1}+1\right)+\left(j_{2}-\ell_{2}\right)\left(2 \ell_{2}+1\right)-L}{\sqrt{6 L(2 L-1)(2 L+1)\left(2 j_{1}+1\right)\left(2 j_{2}+1\right)}} \\
& \times(-1)^{\ell_{2}+j_{2}+1 / 2} \frac{\left(\begin{array}{ccc}
\ell_{1} & \ell_{2} & L \\
1 / 2 & -1 / 2 & 0
\end{array}\right)}{\left(\begin{array}{ccc}
L-1 & j_{1} & j_{2} \\
0 & 0 & 0
\end{array}\right)}
\end{aligned}
$$

and

$$
\begin{aligned}
& \left\{\begin{array}{ccc}
\ell_{1} & \ell_{2} & L \\
j_{1} & j_{2} & L \\
1 / 2 & 1 / 2 & 1
\end{array}\right\}= \\
& \frac{\left(j_{1}-\ell_{1}\right)\left(2 \ell_{1}+1\right)+\left(j_{2}-\ell_{2}\right)\left(2 \ell_{2}+1\right)+L+1}{\sqrt{6(L+1)(2 L+1)(2 L+3)\left(2 j_{1}+1\right)\left(2 j_{2}+1\right)}} \\
& \times(-1)^{\ell_{2}+j_{2}+1 / 2} \frac{\left(\begin{array}{ccc}
\ell_{1} & \ell_{2} & L \\
1 / 2 & -1 / 2 & 0
\end{array}\right)}{\left(\begin{array}{rrr}
L+1 & j_{1} & j_{2} \\
0 & 0 & 0
\end{array}\right)}
\end{aligned}
$$

but in that case $j_{1}+j_{2}+L$ must be odd. In this way, six entries from above mentioned twelve entries of table 10.3 of Ref. [13] are covered with three expressions. Although different approaches are needed for the remaining six entries of Table 10.3 of Ref. [13] with $\ell_{1}-j_{1}=j_{2}-\ell_{2}$, which corresponds to the odd linear combinations of $j_{1}+j_{2}+L^{\prime}$, all twelve tabulated expressions are ratios of the factorized elementary linear functions under the square root. Therefore, it is possi- ble to write the binary recursion relations, for example, which allow to express

$$
\left\{\begin{array}{ccc}
\ell_{1} & \ell_{2} & L+1 \\
j_{1} & j_{2} & L^{\prime}+1 \\
1 / 2 & 1 / 2 & 1
\end{array}\right\}
$$

in terms of

$$
\left\{\begin{array}{ccc}
\ell_{1} & \ell_{2} & L \\
j_{1} & j_{2} & L^{\prime} \\
1 / 2 & 1 / 2 & 1
\end{array}\right\}
$$

separately for $L^{\prime}-L=0, \pm 1$. In order to investigate recursion relations for these $9 j$ symbols taken independently, let us start from the following identity $[13,15,18]$ :

$$
\left\{\begin{array}{lll}
\ell_{1} & \ell_{2} & L \\
j_{1} & j_{2} & L \\
1 / 2 & 1 / 2 & 1
\end{array}\right\}=\frac{\overline{\ell_{1}}+\overline{j_{2}}-\overline{j_{1}}-\overline{\ell_{2}}}{\sqrt{3 \bar{L}}}\left\{\begin{array}{lll}
\ell_{1} & \ell_{2} & L \\
j_{1} & j_{2} & L \\
1 / 2 & 1 / 2 & 0
\end{array}\right\},
$$

which, as mentioned in Ref. [18], was quoted incorrectly in Refs. [14, 22]. Using the expression of the $9 j$ symbol in terms of a $6 j$ symbol [22]:

$$
\left\{\begin{array}{lll}
\ell_{1} & \ell_{2} & L \\
j_{1} & j_{2} & L \\
1 / 2 & 1 / 2 & 0
\end{array}\right\}=\frac{(-1)^{j_{1}+\ell_{2}+L+1 / 2}}{\sqrt{2(2 L+1)}}\left\{\begin{array}{lll}
\ell_{1} & \ell_{2} & L \\
j_{2} & j_{1} & 1 / 2
\end{array}\right\},
$$

we obtain [13]:

$$
\begin{aligned}
& \left\{\begin{array}{lll}
\ell_{1} & \ell_{2} & L \\
j_{1} & j_{2} & L \\
1 / 2 & 1 / 2 & 1
\end{array}\right\}=\frac{(-1)^{j_{1}+\ell_{2}+L+1 / 2}}{\sqrt{6 \bar{L}(2 L+1)}}
\end{aligned}
$$

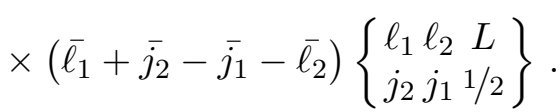

Defining the reduced coefficient

$$
Q_{L}=\sqrt{2 L+1}\left\{\begin{array}{ccc}
\ell_{1} & \ell_{2} & L \\
j_{1} & j_{2} & L \\
1 / 2 & 1 / 2 & 1
\end{array}\right\}
$$

and using the recursion relation for $6 j$ symbols described in Ref. [23], we find

$$
\begin{aligned}
& D_{\ell_{1}, \ell_{2}, j_{1}, j_{2}}(L+1) Q_{L+1}-E_{\ell_{1}, \ell_{2}, j_{1}, j_{2}}(L) Q_{L} \\
& +D_{\ell_{1}, \ell_{2}, j_{1}, j_{2}}(L) Q_{L-1}=0,
\end{aligned}
$$

where

$$
\begin{aligned}
& D_{\ell_{1}, \ell_{2}, j_{1}, j_{2}}(L)= \\
& \left\{\left(L^{2}-1\right)\left[L^{2}-\left(\ell_{1}-\ell_{2}\right)^{2}\right]\left[\left(\ell_{1}+\ell_{2}+1\right)^{2}-L^{2}\right]\right\}^{1 / 2} \times
\end{aligned}
$$




$$
\times\left\{\left[L^{2}-\left(j_{2}-j_{1}\right)^{2}\right]\left[\left(j_{2}+j_{1}+1\right)^{2}-L^{2}\right]\right\}^{1 / 2}
$$

and

$$
\begin{aligned}
& E_{\ell_{1}, \ell_{2}, j_{1}, j_{2}}(L)= \\
& (2 L+1)\left\{\bar{L}\left[-\bar{L}+\ell_{1}\left(\ell_{1}+1\right)+\ell_{2}\left(\ell_{2}+1\right)\right]\right. \\
& +j_{2}\left(j_{2}+1\right)\left[\bar{L}+\ell_{1}\left(\ell_{1}+1\right)-\ell_{2}\left(\ell_{2}+1\right)\right] \\
& +j_{1}\left(j_{1}+1\right)\left[\bar{L}-\ell_{1}\left(\ell_{1}+1\right)+\ell_{2}\left(\ell_{2}+1\right)\right] \\
& -3 \bar{L} / 2\} .
\end{aligned}
$$

It can be better, in the case of large arguments in the $9 j$ symbols, to work with the ratio of consecutive elements [24]

$$
R_{L}=\frac{Q_{L}}{Q_{L-1}},
$$

which obeys the following recursion relation:

$$
R_{L+1}=F_{\ell_{1}, \ell_{2}, j_{1}, j_{2}}(L) R_{L}+G_{\ell_{1}, \ell_{2}, j_{1}, j_{2}}(L),
$$

with

$$
F_{\ell_{1}, \ell_{2}, j_{1}, j_{2}}(L)=\frac{E_{\ell_{1}, \ell_{2}, j_{1}, j_{2}}(L)}{D_{\ell_{1}, \ell_{2}, j_{1}, j_{2}}(L+1)}
$$

and

$$
G_{\ell_{1}, \ell_{2}, j_{1}, j_{2}}(L)=-\frac{D_{\ell_{1}, \ell_{2}, j_{1}, j_{2}}(L)}{D_{\ell_{1}, \ell_{2}, j_{1}, j_{2}}(L+1)} .
$$

The $9 j$ symbol of Eq. (13) can be formulated in terms of two $6 j$ symbols [13]:

$$
\begin{aligned}
& \left\{\begin{array}{ccc}
\ell_{1} & \ell_{2} & L \\
j_{1} & j_{2} & L-1 \\
1 / 2 & 1 / 2 & 1
\end{array}\right\}(-1)^{j_{1}+\ell_{2}+L-1 / 2} \sqrt{6 L\left(4 L^{2}-1\right)}= \\
& \sqrt{\left(L^{2}-\left(j_{1}-j_{2}\right)^{2}\right)\left[\left(j_{1}+j_{2}+1\right)^{2}-L^{2}\right]}\left\{\begin{array}{lll}
\ell_{1} & \ell_{2} & L \\
j_{2} & j_{1} & 1 / 2
\end{array}\right\} \\
& +\sqrt{\left(L^{2}-\left(\ell_{1}-\ell_{2}\right)^{2}\right)\left[\left(\ell_{1}+\ell_{2}+1\right)^{2}-L^{2}\right]}\left\{\begin{array}{lll}
\ell_{1} \ell_{2} & L-1 \\
j_{2} & j_{1} & 1 / 2
\end{array}\right\} .
\end{aligned}
$$

Each of the two $6 j$ symbols involved in the latter identity can be evaluated by recurrence, but we did not find any recursion relation for the $9 j$ symbol itself.

We proposed a recursive calculation for special cases of $9 j$ symbols, encountered, for instance, in the transformation matrices from $L S$ to $j j$ coupling schemes, or in the evaluation of matrix elements of tensor operators. We hope that the recursion relation will be useful for the enumeration and algebraic manipulation of $9 j$ symbols.

\section{References}

[1] Z.B. Rudzikas, Theoretical Atomic Spectroscopy (Cambridge University Press, Cambridge, 1997).

[2] B.R. Judd, Operator Techniques in Atomic Spectroscopy (McGraw-Hill, New York, 1963).

[3] A.P. Jucys and A.A. Bandzaitis, Teoriya Momenta Kolichestva Dvizheniya v Kvantovoi Mekhanike (Angular Momentum Theory in Quantum Mechanics) [in Russian] (1st ed.: Mintis, Vilnius, 1965, 2nd ed.: Mokslas, Vilnius, 1977).

[4] S.J. Ališauskas and A. Jucys, J. Math. Phys. 12, 594 605 (1971). Erratum: J. Math. Phys. 13, 575 (1972).

[5] A.C.T. Wu, J. Math. Phys. 14, 1222-1223 (1973).

[6] L.C. Biedenharn and J.D. Louck, Angular Momentum in Quantum Physics (Addison-Wesley, Reading, MA, 1981).

[7] D. Zhao and R.N. Zare, Mol. Phys. 65, 1263-1268 (1988).

[8] K.S. Rao, V. Rajeshwari, and C.B. Chiu, Comput. Phys. Commun. 56, 231-248 (1989).

[9] L. Wei, Comput. Phys 12, 632-634 (1998).

[10] L. Wei, Comput. Phys. Commun. 120, 222-230 (1999).

[11] G. Gaigalas, T. Žalandauskas, and Z. Rudzikas, Lith. J. Phys. 41, 226-231 (2001).

[12] A.V. Karosiené, S.J. Ališauskas, and A.A. Bandzaitis, Lietuvos Fiz. Rinkinys 5, 13-21 (1965) [in Russian].

[13] D.A. Varshalovich, A.N. Moskalev, and V.K. Khersonskii, Quantum Theory of Angular Momentum (World Scientific, Singapore, 1988).

[14] A. de-Shalit and I. Talmi, Nuclear Shell Theory (Academic, New York, 1963).

[15] L. Szybisz and V. Seshagiri Rao, Lett. Nuovo Cimento 14, 237-240 (1975).

[16] D.M. Brink and G.R. Satchler, Angular Momentum (Clarendon Press, Oxford, 1968).

[17] R.T. Sharp, Nucl. Phys. A 95, 222-228 (1967).

[18] S. Jang, J. Math. Phys. 9, 397-402 (1968).

[19] L.C. Biedenharn, J.M. Blatt, and M.E. Rose, Rev. Mod. Phys. 24, 249-257 (1952).

[20] T. Regge, Nuovo Cimento 10, 544-545 (1958).

[21] M. Nomura, J. Phys. Soc. Jpn. 58, 2677-2686 (1989).

[22] M. Rotenberg, R. Bivins, N. Metropolis, and J.K. Wooten, The 3-j and 6-j symbols (The Technology Press, M. I. T., Cambridge, MA, 1959).

[23] K. Schulten and R.G. Gordon, J. Math. Phys. 16, 19611970 (1975).

[24] J.H. Luscombe and M. Luban, Phys. Rev. E 57, 72747277 (1998). 


\title{
DĖL KAI KURIŲ $9 j$ KOEFICIENTŲ REKURENTINIO SKAIČIAVIMO
}

\author{
J.-C. Pain
}

Atominès energijos ir alternatyviuju energiju komisariatas, Arpajon, Prancūzija

\section{Santrauka}

Judejjimo kiekio momentu perrišimo matricu skaičiavimas gali būti labai ilgas ir čia naudingi alternatyvūs metodai, netgi jei jie tinka tik ypatingais atvejais. Pateikiamas rekurentinis sąryšis skaičiuoti tam tikrus $9 j$ koeficientus, naudojamus judejjimo kiekio momento kvantineje teorijoje. 